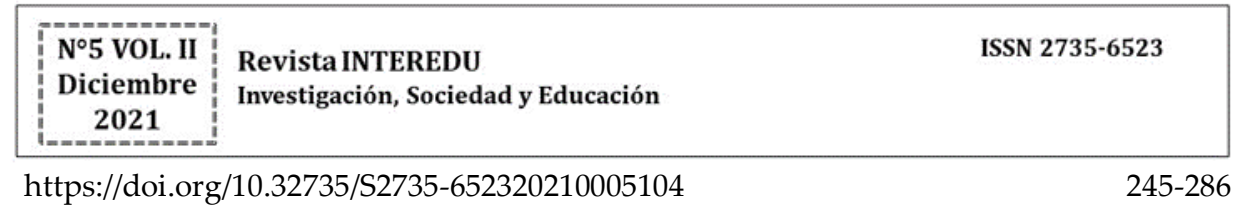

\title{
CONTRIBUCIÓN DE UN PROGRAMA DE FORMACIÓN CONTINUA A LAS CONCEPCIONES EPISTEMOLÓGICAS EN DOCENTES DE QUÍMICA
}

\section{Contribution from continuous training program to the epistemological conceptions in chemistry teachers}

\author{
SANDRA VILLEGAS FERNÁNDEZ \\ Universidad Católica de la Santísima Concepción \\ svillegas@magisteredu.ucsc.cl \\ https://orcid.org/0000-0001-5777-9084 \\ LUIGI CUELLAR FERNÁNDEZ \\ Universidad Católica de la Santísima Concepción \\ lcuellar@ucsc.cl \\ https://orcid.org/0000-0002-0659-9101
}

\section{RESUMEN}

El presente artículo se enmarca en una investigación con un grupo de docentes de Química de educación secundaria chilena que participaron en talleres de formación continua. El objetivo de este estudio fue caracterizar la evolución de las concepciones epistemológicas sobre la naturaleza de las ciencias y la importancia de la fundamentación metadisciplinar para el mejoramiento de las prácticas docentes. A partir de la investigación, de carácter cualitativa con un enfoque descriptivo en un estudio de casos colectivo, queda en evidencia que el conocimiento de las concepciones epistemológicas vinculadas a la naturaleza de las ciencias, en el marco de una comunidad de aprendizaje, permiten resaltar la importancia de promover la reflexión docente y el cambio en su discurso pedagógico.

Palabras clave: Concepciones epistemológicas; naturaleza de las ciencias; formación docente; comunidad de aprendizaje; prácticas docentes. 


\section{ABSTRACT}

This article is part of a research conducted over a group of Chilean secondary-education chemistry teachers who participated in continuing education workshops. The objective of this study was to characterize the evolution of epistemological conceptions about the Nature of Sciences, and the importance of the metadisciplinary foundation for the improvement of teaching practices. This qualitative research of a collective case study has been framed over descriptive approach and revealed that knowledge on epistemological conceptions linked to the Nature of Sciences, within the framework of a learning community, and also allowed to highlight the importance of promoting teacher reflection and a change in its pedagogical discourse.

Key words: Epistemological conceptions; nature of sciences; teacher training; learning community; teaching practices.

\section{INTRODUCCIÓN}

En el campo de la didáctica de las ciencias hay toda una corriente de reflexión que destaca la necesidad de incorporar y vincular las metaciencias a los procesos de formación inicial y continua del profesorado de ciencias (Quintanilla et al., 2006a). Este saber permite relacionar el conocimiento científico en cada momento de la historia y los problemas que se intentan solucionar, los fines que se persiguen, las herramientas conceptuales utilizadas y las metodológicas disponibles, así como la cultura y los valores vigentes en ese momento. De este modo se comprenderá el enorme valor disponible en los contenidos provenientes de estas disciplinas, para orientar la práctica profesional de los profesores y profesoras de ciencias naturales (Quintanilla et al., 2006b). 
Investigaciones desarrolladas por Quintanilla y Ravanal (2009) sobre la formación docente plantean la necesidad de que el profesorado conozca qué es la ciencia, por qué es enseñada, cuál es la naturaleza del conocimiento científico, cómo se adquiere conocimiento conceptual, de qué forma se desarrolla conocimiento procedimental sobre ciencia y cuáles son los métodos o estrategias de enseñanza que facilitan apropiadamente la adquisición de conocimientos científicos en el aula. La evidencia apunta a que una gran parte del profesorado no valora los aspectos epistemológicos que entraña la didáctica de las ciencias como disciplina metacientífica a la hora de fundamentar, planificar y desarrollar sus clases (Quintanilla y Ravanal, 2009).

Por otra parte, si bien existe una preocupación de generar instancias de formación continua y colaboración entre docentes, éstas no se han dado en un escenario que satisfaga totalmente los requerimientos del profesorado, pues, por ejemplo, en Chile las capacitaciones docentes no se han orientado a mejorar las prácticas docentes (Ministerio de Educación de Chile [Mineduc], 2017).

Según Briscoe (1991), en la actualidad los procesos de formación docente están dirigidos al aseguramiento de la calidad en la formación de los futuros pedagogos y con ese fin se han creado programas y becas para apoyar dicha formación. Sin embargo, estas medidas se han orientado principalmente a controlar el ingreso y egreso del proceso de formación inicial de docentes, pero no se han orientado al proceso de formación continua del docente. Plantea Briscoe (1991) que es un hecho bastante conocido que muchos profesores participan en cursos de formación con la intención de perfeccionarse profesionalmente y así enfrentar de mejor manera el proceso formativo, creen de este modo, estar mejor preparados para utilizar nuevas técnicas y nuevos materiales. Sin embargo, una gran 
parte de dichos profesores antes de que puedan darse cuenta acaban enseñando de la misma forma como lo han hecho siempre.

La transcendencia que tiene la formación docente de profesores de ciencias ha permitido que en el último tiempo hayan aumentado considerablemente las investigaciones relacionadas con las concepciones epistemológicas de este profesorado, así como la relación con su práctica docente. En general, estos estudios están referidos al estudio de caracterizaciones de concepciones sobre enseñanza y aprendizaje de la ciencia en docentes de Biología, (Ravanal y Quintanilla, 2010), y de profesores de enseñanza media (Tobin y Campbell, 1997; Carvajal y Gómez, 2002; Fernández et al., 2009; Camacho, 2013).

Sin embargo, de acuerdo a Vildósola (2017), en Chile las investigaciones sobre el conocimiento epistemológico del profesorado de ciencias son aún escasos. Según este autor, el profesorado chileno en ejercicio no tiene el conocimiento ni la comprensión de la epistemología de la ciencia para incorporarla a la enseñanza y práctica de aula. Esta afirmación se constata cuando se visualiza una práctica docente tradicional que enfatiza la transmisión de contenidos de ciencias, sin la adecuada contextualización y muy deficiente en el desarrollo de procesos de indagación (Pavéz y Pérez, 2013). Por otro lado, Acevedo (2004) plantea que es posible evidenciar que el docente de ciencias no tiene, en general, una buena compresión de la naturaleza de las ciencias; por tanto, es evidente la necesidad de prestar atención a este componente metateórico en los cursos de formación del profesorado de ciencias de todos los niveles educativos, tanto durante la formación inicial como posteriormente en el ejercicio profesional.

Adicional a la importancia del conocimiento epistemológico de la ciencia por parte del profesorado, se considera fundamental el conocimiento 248 | INTEREDU № 5 VOL. II (DicieMBRE 2021) PÁGs. 245-286. ISSN: 2735-6523 
pedagógico y el conocimiento didáctico, lo que algunos autores conciben como el esquema conceptual del profesor (Angulo, 2002; Quintanilla, 2006; Cuellar 2010). Respecto al conocimiento didáctico, Martínez y González (2014) plantean una clara debilidad en la formación docente cuando no se considera la formación didáctica, incidiendo en el uso de una mayor variedad de estrategias de enseñanza por parte del docente. Del mismo modo, señalan que cursos relacionados explícitamente con la naturaleza de la ciencia están totalmente ausentes del currículum, lo cual sería negativo ya que una cierta visión epistemológica del conocimiento científico constituye una necesidad en el profesorado de ciencias.

García-Carmona (2012), señala que se hace necesario promover instancias de formación de docentes orientadas a la adquisición de un conocimiento didáctico del contenido para la enseñanza de la naturaleza de las ciencias. De aquí surge la importancia de entender la influencia que puedan tener talleres de formación continua en las concepciones epistemológicas de los docentes, que promueva un análisis reflexivo y crítico de sus prácticas pedagógicas.

En este sentido, el objetivo general de este artículo es caracterizar la evolución de las concepciones epistemológicas sobre naturaleza de las ciencias en docentes que participaron en talleres de formación continua, desde la necesidad de fundamentación metadisciplinar para mejorar las prácticas docentes, a partir de los siguientes objetivos específicos: 1) Identificar las concepciones epistemológicas acerca de la naturaleza de las ciencias en docentes de química que se desempeñan en Educación Media; 2) Comparar las concepciones epistemológicas acerca de la naturaleza de las ciencias de los docentes antes y después de la participación en talleres de formación continua; y 3) Interpretar cómo las concepciones epistemológicas acerca de la naturaleza de las ciencias cambian a partir de la participación de los docentes en talleres de formación continua para el mejoramiento de sus prácticas docentes. 


\section{MARCO TEÓRICO}

En este apartado se presenta una caracterización de la ciencia, su naturaleza y las visiones dogmáticas o racionalistas, así como algunos elementos que han de considerarse en el marco referencial del quehacer de profesor de ciencias. Además, se caracterizan las nociones de didáctica de las ciencias, el conocimiento didáctico del contenido y los procesos de formación docente.

\subsection{LA CIENCIA COMO CUERPO DE CONOCIMIENTO}

Desde el punto de vista de los epistemólogos de la ciencia, Karl Popper (1962) planteaba que la tarea de la ciencia es elaborar proposiciones y sistemas de proposiciones -teorías- acerca del mundo, las cuales debían ser contrastadas de manera empírica. Por su parte, y a partir de la necesidad de justificar el desarrollo de teorías científicas, por otro lado, Kuhn (2019) establece una nueva concepción epistemológica, donde reconoce que el desarrollo del conocimiento científico está determinado por la existencia de paradigmas de competencia, y que los miembros de las comunidades científicas comparten un paradigma, proponiendo y solucionando problemas dentro de una comunidad de saber.

Frente a estas posturas, Imre Lakatos (1983) propone el concepto de programas de investigación científica, caracterizados por la presencia de un núcleo firme, que se protege de las refutaciones mediante un cinturón protector de hipótesis auxiliares, y presenta una heurística capaz de solucionar los problemas e incluso convirtiéndolos en evidencias positivas.

En este estudio, los autores adhieren a los planteamientos de Stephen Toulmin (1977) quien en su crítica al positivismo lógico, a las posturas absolutistas y al radicalismo de los revolucionistas, asumen que las teorías 250 | INTEREDU № 5 VOL. II (DiCIEMBRE 2021) PÁGs. 245-286. ISSN: 2735-6523 
científicas deben ser analizadas en su proceso de desarrollo, lo que involucra -entre otros aspectos- tener en cuenta la dimensión individual y colectiva de los conceptos, los cuales junto a las teorías y los procedimientos están en evolución constante, siendo objeto de pequeños cambios.

Esta postura sobre la ciencia nos lleva entonces a entender una dinámica de constante cambio y renovación, una evolución permanente de los conceptos científicos. Esta imagen de la ciencia nos debiera entonces remitir a posturas análogas respecto a las ideas de la ciencia que se enseña en el aula escolar.

\subsection{ENSEÑANZA DE LAS CIENCIAS}

Desde la visión clásica de la enseñanza de las ciencias, los problemas que debe resolver el docente son más bien de tipo instrumental. Desde este paradigma, se considera que se debe capacitar al docente para programar y evaluar. En esta programación se fijan los objetivos por alcanzar, estableciendo los logros de los estudiantes en términos de conductas observables. En este sentido, esta planificación se lleva en forma muy rigurosa, dejando en segundo plano las necesidades específicas de los estudiantes, pues se centran en la entrega de contenidos conceptuales (Aiello, 2004).

Esta postura se basa en una visión absolutista y dogmática del conocimiento científico, así como en una enseñanza basada en la transmisión de conocimientos. A los contenidos del currículum y a la forma en que se enseña se le presta poca atención.

La manera como se conciben los conceptos enseñar y aprender, educar y formar, centra la preocupación en enseñar procedimientos, protocolos y verdades, y deja de lado otras formas de interpretar la realidad, el saber y las ciencias. No se da espacio a reflexionar sobre las dudas y expectativas que puedan surgir en el estudiante más allá de lo INTEREDU № 5 VOL. II (DiCIEMBRE 2021) PÁGs. 245-286. ISSN: 2735-6523| 251 
que el currículum educacional le ofrece (Aldana, 2008; Ravanal y Quintanilla, 2010; Martínez, 2015).

Desde la visión post-positivista de la ciencia, hay una preocupación por las concepciones alternativas, las ideas previas o teoría implícitas de los alumnos en la enseñanza de las ciencias. Desde este paradigma, el estudiante se debe enfrentar en su aprendizaje al difícil proceso de cambio conceptual, entendido como el proceso por el cual modifica sus concepciones o teorías personales. Este cambio conceptual reviste especial importancia en la revisión de las propias concepciones de los docentes (Aiello, 2004).

En este sentido, está claro que la enseñanza de la ciencia necesita de un docente que fundamente su actuar en el aula, con una clara visión epistemológica, coherente con los desafíos de la educación actual y con las necesidades de sus estudiantes. Por tanto, la imagen de ciencia que los profesores poseen, así como sus concepciones epistemológicas, son importantes de reconocer e identificar y cómo estas se relacionan con su quehacer profesional (Ravanal et al., 2012).

Quintanilla (2006), señala que enseñar ciencias, es mucho más que enseñar contenidos y presentar temas de las ciencias. El autor plantea que si la ciencia es asumida como una disciplina que aborda una manera de entender el universo -sea en esferas globales o locales-, la enseñanza de las ciencias debe atender, también, los aspectos para el desarrollo de formas de comprender el mundo que habitamos, así como los modos de construir conocimientos en las ciencias. De aquí se entiende que la enseñanza de los procesos de construcción del conocimiento sea un objetivo a ser puesto en práctica en las aulas.

Desde un punto de vista normativo, el Mineduc (2017) sostiene como premisa, que la enseñanza y aprendizaje de la ciencia es un proceso activo en el cual la investigación y la resolución de problemas ocupan un sitial importante. 252 | INTEREDU № 5 VOL. II (DicIEMBRE 2021) PÁGs. 245-286. ISSN: 2735-6523 
Rodríguez y López (2006), señalan que este aprendizaje permite que los estudiantes conozcan cómo se ha construido el conocimiento científico, comprender que la ciencia es un conocimiento universal que evoluciona a través del tiempo, que está expuesto a nuevas evidencias y que, por lo tanto, es igualmente un saber dinámico.

En este sentido, está claro que existe una necesidad de identificar las concepciones epistemológicas, referidas a la Naturaleza de la ciencia según los docentes para luego comprender cómo una intervención, como sería la participación en talleres de formación continua, puede contribuir al cambio o fortalecimiento en estas concepciones.

\subsection{NATURALEZA DE LA CIENCIA}

Aunque la preocupación por la comprensión de la naturaleza de la ciencia como objetivo de la educación viene desde comienzo de siglo, la realidad es que ha estado poco presente en los currículos escolares durante muchos años, $y$, en consecuencia, también en la formación general del profesorado (Lederman, 1992, citado por Alonso, 2000). La naturaleza de la ciencia se concibe como un conjunto de contenidos metacientíficos con valor para la educación científica, con la finalidad de promover reflexión, posición epistemológica y mejorar la enseñanza y aprendizaje de los contenidos científicos (Adúriz-Bravo, 2007).

Por otro lado, autores como Tamayo y Orrego (2009) señalan que son importantes los lazos establecidos entre el conocimiento de la naturaleza de la ciencia y la habilidad de los profesores para implementar modelos de cambio y evolución conceptual en el aula. En la misma línea, Lederman (2006) plantea que el valor investigativo de las concepciones epistemológicas radica en dos ideas fundamentales: la comprensión de la naturaleza de la ciencia del profesorado guarda cierta relación con la de sus estudiantes y la imagen que estos adquieren de la ciencia; y las creencias del profesorado sobre naturaleza 
de la ciencia influyen significativamente en la forma de enseñar ciencia y en las decisiones que se toman en el aula.

Bajo esta mirada, se debe tener en cuenta que las concepciones de ciencia son indisolubles de la práctica de un docente y constituyen una fuente de información que podría aportar al cambio de las prácticas educativas del profesorado.

Para los efectos del objetivo del presente artículo, se hace indispensable realizar un acercamiento a una reflexión epistemológica contraria al dogmatismo, que favorezca a la construcción de una imagen de la ciencia realista y racionalista moderada (Ravanal y Quintanilla, 2010). Desde un enfoque tradicional surge la concepción dogmático-positivista de la ciencia y su enseñanza, mientras que por otro, existe una concepción de carácter racionalista moderada cercana al constructivismo. Es justamente desde el campo de la investigación en la enseñanza de las ciencias que estos temas toman relevancia, por lo que es fundamental plantear un acercamiento a una de las ciencias de la enseñanza: la didáctica de las ciencias.

\subsection{LA NATURALEZA DE LA CIENCIA EN LOS DOCENTES: DOGMATISMO} Y RACIONALISMO, DOS POSTURAS DIVERGENTES.

Solbes y Torres (2013) definen las concepciones como -organizadores implícitos de los conceptos, de naturaleza esencialmente cognitiva y que incluyen creencias, significados, conceptos, proposiciones, reglas, imágenes mentales, preferencias, entre otros-, que influyen en lo que se percibe y en los procesos de razonamiento que se realizan. Por otro lado, Martínez y González (2014), señalan que las concepciones son construcciones mentales que buscan proporcionar explicaciones de los fenómenos, interactuando el conocimiento formal e informal. Las concepciones 
epistemológicas de los docentes poseen una relevancia fundamental en el estudio de su configuración mental pues permiten comprender el porqué de su postura, su pensamiento y su acción en el aula de clase (Salazar y Serrano, 2017).

Alburquerque (2016) explica al dogmatismo positivista como postura epistemológica según la cual el conocimiento es simplemente posible. Según el autor, el dogmatismo epistemológico da como un hecho la posibilidad del conocimiento, pero ello nos muestra que el conocimiento desde esta perspectiva es más bien una aspiración antes que una verdad objetiva, pues no logra ver que el conocimiento es más bien una relación entre sujeto y objeto.

Una concepción dogmático positivista se conecta con aquellas ideas de ciencia en la que esta permanece estática, incuestionable, caracterizada por la orientación tradicional de las actividades de enseñanza y aprendizaje (Izquierdo, 2005). Por su parte, en el caso del racionalismo, la forma de acceder al conocimiento es por medio de la razón. El ser humano posee ideas innatas y cuando pensamos, proyectamos esas ideas a la comprensión de un hecho, los cuales tienen múltiples fases, pero sólo podemos capturar una de ellas (Alarcón, 2018).

Una concepción racionalista-moderada de la ciencia se distancia notoriamente de perspectivas más tradicionales, por lo que la idea e imagen de ciencia se asocia a una disciplina en constante transformación, respecto a cómo se construye y evoluciona el conocimiento científico. Desde esta concepción, se considera relevante utilizar y promover la intuición, la imaginación y creatividad para la interpretación de los fenómenos naturales de nuestro entorno, así como considerar las ideas o representaciones previas de fenómenos naturales (Izquierdo, 2005).

Adúriz-Bravo (2004), señala la pertinencia de tener en cuenta las epistemologías de los docentes, como condición para identificar y 
favorecer cambios didácticos en la enseñanza de las ciencias. Sin una verdadera transformación conceptual sobre la esencia del aprendizaje, la práctica del docente enfrentará contradicciones, al aplicarse estrategias que limitan las posibilidades de adoptar enfoques alternativos en la construcción de conocimiento.

Lederman (2006) plantea que las concepciones de los docentes de ciencias en estos temas afectan de manera directa a las concepciones de los estudiantes e influyen en la conducta de los docentes y en el desarrollo de su práctica. Por otro lado, las concepciones de los docentes pueden evolucionar en función de la construcción y reestructuración de nuevos conocimientos a partir de la interacción y reflexión de ideas y experiencias a partir de una efectiva formación docente dentro de una comunidad de aprendizaje (Porlán et al., 1998). Por tanto, el estudio de las concepciones epistemológicas de los docentes cobra especial importancia al momento de fortalecer los procesos de formación docente.

\section{MetodologíA}

La investigación que se reporta en este artículo se desarrolló mediante una metodología de tipo mixto con un enfoque mayormente interpretativo mediante el estudio de caso colectivo. El enfoque interpretativo permite especificar las características y perfiles de las personas, grupos o comunidades o cualquier fenómeno que se someta a un análisis (Hernández y Torres, 2018). Por su parte, el estudio de caso colectivo, permite un análisis sistemático y en profundidad. Bisquerra y Alzina, (2004) señalan que el estudio de casos interpretativo aporta descripciones profundas y valiosas con el fin de interpretar y teorizar sobre el caso. En el artículo, este fenómeno colectivo 
corresponde a las características surgidas a partir de percepciones entregadas por los docentes.

Esta investigación pretendió aportar con sus resultados a la discusión respecto de la necesidad de reflexionar acerca de la manera en que se concibe la enseñanza de las ciencias y valorar que a través de talleres de formación continua se lograran instancias de enriquecimiento de su discurso teórico. Por tanto, el objetivo de este estudio fue caracterizar la evolución de las concepciones epistemológicas sobre naturaleza de la ciencia en los docentes que participaron de talleres de formación continua desde la necesidad de fundamentación metadisciplinar para el mejoramiento de las prácticas docentes.

\subsection{MUESTRA}

La muestra la constituyeron seis docentes de Química en ejercicio de la provincia de Concepción, resguardando el cumplimiento de criterios de voluntariedad, diversidad en la dependencia del establecimiento, años de servicio y que se desempeñaran en distintos niveles educacionales.

Tabla 1. Caracterización de los participantes del estudio.

\begin{tabular}{llll}
\hline Docente & $\begin{array}{c}\text { Dependencia del } \\
\text { establecimiento } \\
\text { educacional }\end{array}$ & Formación inicil de pregrado & $\begin{array}{l}\text { Años de } \\
\text { servicio } \\
\text { docente }\end{array}$ \\
\hline 1 & Municipal & Pedagogía en CCNN y Química & 10 \\
\hline 2 & Particular & Pedagogía en Biología y Química & 23 \\
\hline 3 & Particular & Pedagogía en CCNN y Química & 3 \\
\hline 4 & Municipal & Pedagogía en Química & 19 \\
\hline 5 & Particular & Pedagogía en CCNN y Química & 5 \\
\hline 6 & Particular & Pedagogía en Biología y Química & 22 \\
\hline
\end{tabular}

Fuente: Elaboración propia. Nota: $\mathrm{CCNN}=$ Ciencias Naturales 
Los docentes participaron durante un año de 57 sesiones de talleres de formación continua, como parte del proyecto de investigación FONDECYT 11150509. Las temáticas abordadas fueron: concepto de ciencia, didáctica de las ciencias y aportes a las disciplinas metateóricas; epistemología de las ciencias; naturaleza de las ciencias; competencias escolares; comunidades de aprendizaje; cambio didáctico en el docente; e historia de las ciencias y cuestiones sociocientíficas. Sin embargo, para la recolección de datos se consideraron 17 de las sesiones, las cuales eran pertinentes al objetivo que dirigió este estudio.

\subsection{INSTRUMENTOS DE RECOLECCIÓN DE INFORMACIÓN}

A continuación, se describen brevemente los instrumentos que permitieron la recolección de información para este estudio.

\subsubsection{CUESTIONARIO LIKERT}

El instrumento utilizado ha sido un cuestionario en formato de escala Likert, desarrollado por Quintanilla et al. (2010). El instrumento considera ocho dimensiones de análisis: naturaleza de la ciencia, enseñanza de las ciencias, aprendizaje de las ciencias, evaluación de los aprendizajes científicos, rol del profesor de ciencias naturales, resolución de problemas científicos, competencias de pensamiento científico y planificación de la enseñanza). En cada dimensión se proponen diez enunciados que representan dos visiones epistemológicas antagónicas: dogmática-positivista y racionalista-moderada. Para efectos del objetivo de este estudio, solo se consideraron los resultados para la dimensión de la naturaleza de la ciencia, cuyos ítems están ligados a las concepciones epistemológicas de los docentes. 

docentes de química

El instrumento utilizado fue sometido originalmente a un proceso de determinación de validez por parte de ocho especialistas en investigación en didáctica en Iberoamérica y por parte de un grupo piloto de 20 profesores de ciencias. A partir de esta valoración, se observaron acuerdos en cuanto a la pertinencia de cada ítem dentro de la dimensión, así como en relación a la claridad de la formulación de los enunciados y uso del lenguaje. En un proceso posterior de análisis estadístico, el instrumento fue ajustado (Quintanilla et al., 2020), arrojando un alfa de Cronbach de 0.889 .

\subsubsection{PRegunta Abierta (COMPlementaria Al CUESTIONARIO} LIKERT)

Para la recolección de información sobre cuál es el rol que los docentes de Química le otorgan al estudio de la naturaleza de las ciencias, se les solicitó a los docentes responder una pregunta abierta antes y después de la participación en los talleres de formación continua, con el propósito de complementar las respuestas al cuestionario.: ¿Cuál es el papel de los estudios sobre la naturaleza de la ciencia para el profesorado de Química? La aplicación de esta pregunta tenía como fin comparar las concepciones epistemológicas acerca de la Naturaleza de las Ciencias de los docentes antes y después de la participación en talleres de formación continua (objetivo específico 2).

\subsubsection{GRUPOS FOCALES}

Esta modalidad de entrevista grupal es abierta y estructurada, generalmente toma la forma de una conversación grupal, en la cual el investigador plantea algunas temáticas -preguntas asociadas a algunos antecedentes que orientan la dirección de la misma-, de acuerdo con los 
propósitos de la investigación (Aigneren, 2002). El grupo focal es la única herramienta a disposición del evaluador que sirve a la vez para analizar y para confrontar la información de los participantes de la muestra. Este instrumento ayuda a comprender la actitud de los participantes, su comprensión y su percepción de una intervención, lo que no sería posible partiendo de una entrevista individual. (Juan y Roussos, 2010).

Se realizaron dos grupos focales por los investigadores, quienes cumplieron el rol de entrevistadores antes y después de la participación de los docentes en talleres de formación continua. Se desarrollaron temas como: naturaleza de las ciencias, aprendizaje y enseñanza de las ciencias, competencias científicas y comunidades de aprendizaje.

Con el fin de identificar y comparar las concepciones epistemológicas acerca de la naturaleza de las ciencias en docentes de química antes y después de la participación en talleres de formación continua (objetivos específicos 1 y 2) se realizaron preguntas como: ¿Por qué es importante conocer las concepciones epistemológicas acerca la naturaleza de la ciencia de los docentes?, ¿Es posible un cambio en las concepciones epistemológicas acerca de la naturaleza de las ciencias en los docentes?, ¿Existe relación entre el conocimiento de la naturaleza de las ciencias y el diseño de propuestas de enseñanzas?, ¿Cuál es el rol de la naturaleza de las ciencias en la contextualización en la enseñanza?, ¿Cuál es el rol de la epistemología frente a nuevos conocimientos?, ¿Cuál es el aporte de una comunidad de docentes en la práctica pedagógica?

Se debe recalcar que los seis docentes que integraron la muestra autorizaron su participación en este estudio mediante la firma de un consentimiento informado. 


\section{ANÁLISIS DE DATOS}

Para poder identificar y describir las concepciones de los docentes antes y después de la experiencia de los talleres de formación continua, se usaron dos técnicas complementarias, el análisis cuantitativo y cualitativo descriptivo y el análisis de contenido. A pesar de que la primera técnica pertenece a un enfoque cuantitativo, para los efectos de este estudio se pretendió identificar e interpretar concepciones o ideas de los participantes, por lo que su orientación es más bien descriptiva y fundamentalmente se orienta hacia una interpretación cualitativa. Con esta técnica se intentó describir e interpretar las tendencias epistemológicas dogmática positivista o racional moderada de los docentes.

Por otro lado, para identificar y caracterizar las concepciones de los docentes sobre el rol que desempeña el estudio sobre la naturaleza de las ciencias se realizó un análisis de contenido, que es una técnica idónea para interpretar concepciones, ya que esta permite adentrarse en las reflexiones personales respecto de cómo concibe la ciencia un grupo determinado. Por tanto, en general el conjunto de técnicas utilizadas en el presente estudio pertenecen al campo del análisis de contenido, buscando sobre todo interpretar y ordenar el contenido de los mensajes comunicativos de textos, sonidos e imágenes (Abela, 2002). 


\subsection{DIMENSIONES DEL CUESTIONARIO TIPO LIKERT, DIMENSIÓN}

\section{NATURALEZA DE LAS CIENCIAS}

Tabla 2. Enunciados identificados por concepciones dogmática positivista (DP) y racionalista moderado (RM).

\begin{tabular}{|c|c|c|}
\hline \multicolumn{3}{|r|}{ Dimensión: Naturaleza de las ciencias } \\
\hline 1 & $\mathrm{RM}$ & $\begin{array}{l}\text { La metodología científica permite al investigador en ciencias utilizar la intuición y la } \\
\text { imaginación en cualquier momento del proceso de construcción científica. }\end{array}$ \\
\hline 2 & DP & $\begin{array}{l}\text { El estudiante debe aprender la metodología de investigación científica basada en } \\
\text { etapas sucesivas y jerárquicas, rigurosamente planificadas. }\end{array}$ \\
\hline 3 & $\mathrm{RM}$ & $\begin{array}{l}\text { Las ciencias tienen carácter experimental, por ello es indispensable que los } \\
\text { estudiantes construyan los hechos científicos, a partir de los hechos del mundo. }\end{array}$ \\
\hline 4 & $\mathrm{RM}$ & $\begin{array}{l}\text { Los criterios que poseen las ciencias son parciales, porque los hechos de la naturaleza } \\
\text { están sujetos a interpretaciones individuales y sociales. }\end{array}$ \\
\hline 5 & $\mathrm{DP}$ & $\begin{array}{l}\text { La objetividad de los científicos y sus métodos permiten que la ciencia sea neutral e } \\
\text { imparcial frente a la interpretación de los fenómenos del mundo. }\end{array}$ \\
\hline 6 & RM & $\begin{array}{l}\text { Los profesores deben adoptar un modelo de ciencia y de enseñanza de las ciencias, } \\
\text { epistemológicamente fundamentado en una teoría del conocimiento. }\end{array}$ \\
\hline 7 & DP & $\begin{array}{l}\text { Los profesores deben enseñar el conocimiento verdadero, confiable, definitivo e } \\
\text { incuestionable, que se produce en la comunidad científica. }\end{array}$ \\
\hline 8 & $\mathrm{DP}$ & $\begin{array}{l}\text { Las ciencias son rigurosas, ya que, bajo criterios sumamente claros y precisos, los } \\
\text { científicos seleccionan y presentan un determinado modelo del mundo. }\end{array}$ \\
\hline 9 & $\mathrm{DP}$ & $\begin{array}{l}\text { El cambio de una teoría científica por otra se basa en criterios objetivos: prevalece la } \\
\text { que explica mejor el conjunto de fenómenos a que se refiere. }\end{array}$ \\
\hline & $\mathrm{DP}$ & $\begin{array}{l}\text { Los conocimientos científicos que han adquirido un reconocimiento y legitimación } \\
\text { universal, difícilmente cambian. }\end{array}$ \\
\hline
\end{tabular}

Fuente: Cuestionario tipo Likert, elaborado por Quintanilla et al. (2010).

\subsection{CATEGORÍAS DE ANÁLISIS}

Primeramente, se realizó la identificación y selección de las categorías de análisis que configuraban las concepciones de los docentes participantes del estudio, para luego dar la caracterización de cada categoría seleccionada. Como segundo paso, se relacionaron las 
categorías dadas con su presencia en los diversos momentos de la investigación.

\subsection{IDENTIFICACIÓN Y SELECCIÓN DE LAS CATEGORÍAS DE ANÁLISIS DE} LOS DOCENTES PARTICIPANTES DE LA INVESTIGACIÓN EN RELACIÓN A LAS CONCEPCIONES EPISTEMOLÓGICAS.

Con el fin de aclarar los puntos de referencia que fueron el sustento de la elección y análisis del contenido en el discurso de los docentes participantes de la investigación se realizó la identificación y caracterización de aquellas categorías que surgen en cada uno de los momentos de la investigación. Se seleccionaron 4 categorías que son las que se encontraron presentes en su totalidad en los grupos focales (ver Tabla 3).

Tabla 3. Categorías presentes en cada instrumento de recolección de datos.

\begin{tabular}{l}
\hline \\
\cline { 3 - 4 }
\end{tabular}




\subsubsection{RELACIÓN ENTRE LAS CONCEPCIONES ACERCA DE LA NATURALEZA} DE LAS CIENCIAS EN EL DOCENTE Y SU PLANIFICACIÓN DE AULA

Durante estos años el proceso de planificación de los docentes se ha convertido en una labor administrativa. Los profesores nos hemos visto sumergidos en una vorágine de exigencias por parte de los estamentos superiores que poco o nada tienen que ver con una enseñanza basada en los intereses y las competencias de los estudiantes.

De acuerdo a Acevedo, (2010), la naturaleza de la ciencia se considera un contenido esencial en muchos documentos curriculares actuales de enseñanza de las ciencias, e incluso se le ha prestado atención en las recientes evaluaciones internacionales de la alfabetización y competencia científica como, por ejemplo, PISA (2006). Sin embargo, Lederman, (2006), señala que la realidad es que una enseñanza de la naturaleza de la ciencia de calidad y un aprendizaje efectivo de la naturaleza de la ciencia aún están muy lejos de haber calado en la enseñanza de las ciencias.

Con base en lo planteado, en esta categoría se consideraron las evidencias que dieron cuenta del rol que juega, en los docentes, sus concepciones epistemológicas sobre naturaleza de las ciencias a la hora de planificar y organizar sus prácticas pedagógicas. Además, se consideraron dentro de este ámbito aquellas evidencias que nos permitieron identificar la necesidad y la importancia, para los docentes, de establecer una relación entre epistemología, enseñanza y aprendizaje de las ciencias. 
4.3.2. NATURALEZA DE LAS Ciencias COMO EJE EPISTEMOLÓGico PARA EL DESARROLLO DE HABILIDADES Y CONOCIMIENTO CIENTÍFICO.

La naturaleza de las Ciencias Naturales contenidas en la situación de enseñanza de la filosofía de la ciencia es entendida como reflexión epistemológica sobre el conocimiento científico. Permite analizar la capacidad del ser humano de producir conocimientos, además de tener control sobre los procesos físicos, químicos y biológicos del universo y su relación con los procesos culturales (Sánchez y Gómez, 2013).

Izquierdo (2005) recalca la creencia sobre la naturaleza de la ciencia, como componente curricular emergente, que ayudaría a desarrollar en las clases de ciencias naturales una genuina actividad científica escolar en la cual se pongan en marcha diferentes procesos cognitivos y lingüísticos superiores, que pueden contribuir al desarrollo de potentes competencias de pensamiento científico, y al mismo tiempo desarrollar aprendizajes superiores que faciliten en el estudiantado el pensar con teoría los hechos del mundo.

Por ello, es importante reflexionar sobre la naturaleza de la enseñanza de las Ciencias Naturales para poder dar sentido y relevancia didáctica al desarrollo de las competencias científicas en los estudiantes. De esta manera, se considera que la enseñanza de las Ciencias Naturales debe ser asumida con gran responsabilidad, teniendo en cuenta la diversidad de implicaciones didácticas y curriculares en los procesos de producción y apropiación de conocimientos (Sánchez y Gómez, 2013).

En esta categoría se consideraron las evidencias que dan cuenta de la relación entre la comprensión de la naturaleza de las ciencias y la toma de decisiones de los docentes frente al desarrollo de habilidades de sus estudiantes. Para estos efectos, se consideraron las evidencias que demostraron la importancia de la reflexión sobre la naturaleza de las ciencias 
para dar sentido e importancia al desarrollo de competencias científicas en los estudiantes, que propicien la generación de nuevos conocimientos.

\subsubsection{CONOCIMIENTO DE LA DIDÁCTICA Y LA NATURALEZA DE LAS} CIENCIAS POR PARTE DE LOS DOCENTES.

En los últimos veinte años surge y se consolida dentro de la didáctica de las ciencias experimentales una extensa y pujante área de investigación, innovación, docencia y extensión, área que comparte el nombre con su objeto de reflexión teórica: la naturaleza de la ciencia. El reconocimiento de la naturaleza de la ciencia como núcleo importante de la enseñanza de las ciencias comienza también a producirse en otros campos profesionales cercanos a esta disciplina: especialistas en las llamadas metaciencias (Filosofía, Historia, Sociología y Psicología de la Ciencia, entre otras) (Adúriz-Bravo e Izquierdo, 2002).

La naturaleza de las ciencias, en su duplicidad de reflexión didáctica y de contenido a enseñar, surge como respuesta a una serie de finalidades que se engloban comúnmente bajo el nombre genérico de alfabetización científica. De acuerdo a esto, Acevedo (2004) plantea que una ciudadanía científicamente alfabetizada sería capaz de logros como: dar sentido a los fenómenos naturales por medio de modelos teóricos; tomar decisiones informadas en asuntos socio científicos relevantes; incorporar competencias tales como el pensamiento crítico, valorar las ciencias como producto cultural humano, entre otros. Aunque la preocupación por la comprensión de la naturaleza de las ciencias como objetivo de la educación viene desde hace tiempo, se ve poco presente en el currículo nacional y en la formación inicial y continua del profesorado. En relación a lo planteado, se consideró dentro de esta categoría aquellas 
evidencias en el discurso de los docentes que refieren al proceso de reflexión sobre la naturaleza de las ciencias, su funcionalidad y la tarea de enseñar ciencias en el aula. También se tomó en cuenta cuando el propio docente es capaz de cuestionar su formación en didáctica y expresa la necesidad de sustento teórico frente a la enseñanza de las ciencias.

\subsubsection{ENRIQUECIMIENTO DEL DISCURSO DOCENTE A PARTIR DE LA} INTERACCIÓN EN UNA COMUNIDAD DE APRENDIZAJE

O 'Sullivan (2007, citado por Eirín-Nemiña, 2018), define a la comunidad de aprendizaje como una entidad que se hace a sí misma, compuesta por las relaciones mantenidas en el tiempo entre sus miembros y por las soluciones que aportan a los problemas que les preocupan. Los integrantes definen y desarrollan internamente lo que consideran importante de su práctica y su profesión. Por otro lado, Eirín-Nemiña (2018) señala que una comunidad de aprendizaje existe porque se genera un aprendizaje y unas prácticas compartidas entre los miembros. En el caso de los profesores, son lugares formales e informales en los que se dan intercambios de ideas, acciones y actuaciones sobre la naturaleza de su trabajo con sus estudiantes.

En este sentido, se deben generar espacios de discusión y reflexión entre docentes que poseen intereses y comparten problemas comunes, que le permitan enriquecer su conocimiento en modelos teóricos educativos actuales con el fin de mejorar sus prácticas en el aula y orientada hacia la formación de un sujeto competente en Ciencias. Quintanilla (2014) menciona que parte de esta mejora significa integrarse y contribuir a una comunidad en la que el aprendizaje significa una mejora continua de las prácticas docentes y el atraer a personas comprometidas como nuevos miembros. 
Según lo anterior, en esta categoría se consideraron las evidencias que dan cuenta de un docente reflexivo, que manifiesta como aprenden sus estudiantes y que es conocedor de teorías epistemológicas sobre aprendizaje. De igual forma, se tomó en cuenta aquellas evidencias que hablan del mejoramiento continuo de sus prácticas docentes, así como la integración y contribución de cada miembro en un aprendizaje significativo.

\section{RESUltados}

5.1. ANÁLISIS COMPARATIVO DEL CUESTIONARIO LIKERT PRE Y POST DESARROLLO DE TALLERES DE FORMACIÓN CONTINUA DEL GRUPO QUE FORMA PARTE DE LA INVESTIGACIÓN

A continuación, se presentan los resultados referidos a la dimensión naturaleza de las ciencias ( $\mathrm{NdeC}$ ) tanto en el primer cuestionario (C1), aplicado antes de los talleres de formación continua y el segundo cuestionario (C2), aplicado después de los talleres de formación continua.

Tabla 4. Cuadro resumen de resultados Cuestionario Likert, visiones de racionalismo moderado (RM) y dogmatismo positivista (DP) para NdeC, por docente para cuestionario C1 y C2.

\begin{tabular}{ccccc}
\hline & \multicolumn{2}{c}{$\begin{array}{c}\text { \% Racionalidad } \\
\text { moderada }\end{array}$} & \multicolumn{2}{c}{$\begin{array}{c}\text { \% Dogmatismo } \\
\text { Positivista }\end{array}$} \\
\cline { 2 - 5 } Docente & $\mathbf{C 1}$ & $\mathbf{C 2}$ & $\mathbf{C 1}$ & $\mathbf{C 2}$ \\
\hline D1 & 55 & 72.5 & 45 & 27.5 \\
\hline D2 & 60 & 62.5 & 40 & 37.5 \\
\hline D3 & 57.5 & 72.5 & 42.5 & 27.5 \\
\hline D4 & 47.5 & 70 & 52.5 & 30 \\
\hline D5 & 55 & 75 & 45 & 25 \\
\hline D6 & 42.5 & 62.5 & 57.5 & 37.5
\end{tabular}

Fuente: Elaboración propia

268 | INTEREDU № 5 VOL. II (DicieMbre 2021) PÁGs. 245-286. ISSN: 2735-6523 
En la Tabla 4 se visualiza un aumento en los porcentajes de visión Racionalismo Moderado y una disminución de la visión Dogmática positivista para la Naturaleza de las ciencias en todos los docentes. Sobre el análisis estadístico descriptivo de los resultados obtenidos en el pre y post cuestionario Likert, se constatan algunas diferencias y cambios significativos en la concepción de la naturaleza de las ciencias de los docentes participantes. En el siguiente Gráfico, se evidencia la tendencia general de cambio hacia una visión epistemológica Racionalista moderado, que evidencian los docentes a partir de su participación en los talleres de formación continua dentro de una comunidad de aprendizaje (Gráfico 1).

Gráfico 1. Contraste entre el pre (C1) y post (C2) cuestionario tipo Likert sobre la naturaleza de las ciencias.

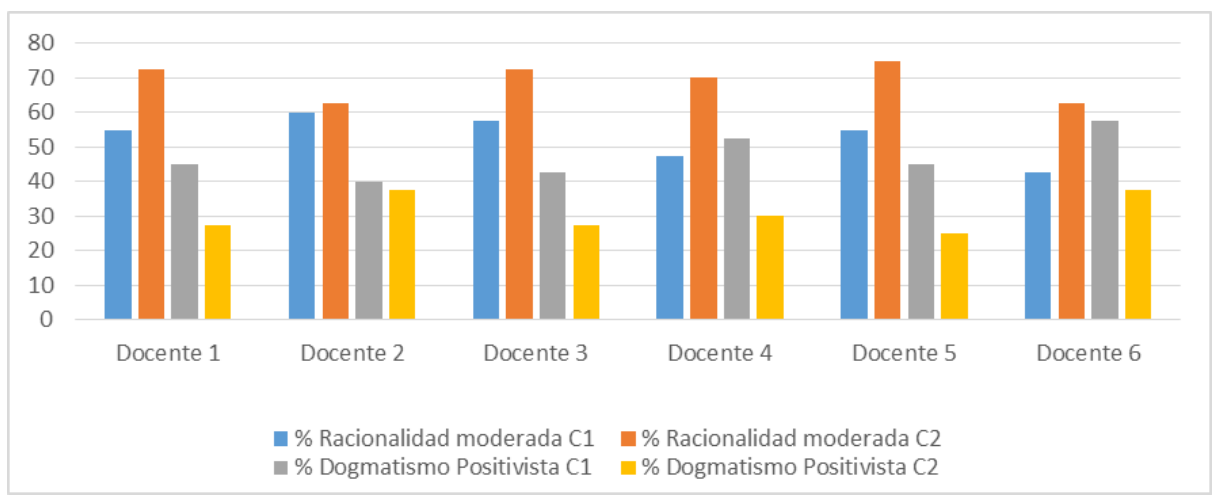

Fuente: Elaboración propia.

En el Gráfico 1 se presenta el contraste entre el pre y post cuestionario, evidenciándose que coexisten las visiones dogmáticas y racionalistas en los docentes tal como revela en resultados otras 
investigaciones relacionadas con el tema de la investigación (Quintanilla y Ravanal 2009; Quintanilla et al., 2010; Ravanal y Quintanilla, 2010).

Considerando los enunciados propuestos en el cuestionario Likert (Tabla 3) y los resultados obtenidos (Tabla 4), se puede mencionar que se evidenció una tendencia marcada de algunos profesores hacia una visión racionalista moderada (D1, D4 y D6). Para estos docentes, las ciencias tienen un carácter experimental, esto se traduce en la necesidad que los estudiantes construyan hechos científicos a partir de los hechos del mundo. Sin embargo, también coinciden en que los docentes son los que enseñan el conocimiento verdadero definitivo e incuestionable (D1 y D2 y D6).

Por otra parte, los resultados arrojaron que existió un alto porcentaje de docentes que están de acuerdo que los profesores deben adoptar un modelo de ciencia que esté cimentado en una teoría del conocimiento. La mayoría está de acuerdo con una visión más post positivista de la ciencia en general, por ejemplo, la relación entre los hechos del mundo y los hechos científicos, dejando de lado la visión dogmática relacionada con la metodología de investigación científica, donde existe un desacuerdo parcial o total con respecto al carácter riguroso y jerárquico y sistemático del conocimiento científico.

Por otro lado, no existe una homogeneidad entre los profesores en reconocer el valor de la intuición y la imaginación en los procesos de construcción científica, donde existe docentes que están de acuerdo (D1, D2, D4, y D6) y otros en desacuerdo (D3 y D5).

También existe una tendencia hacia el dogmatismo (aún después de los talleres de formación continua) al considerar que los científicos bajo criterios precisos, seleccionan y presentan un determinado modelo de mundo. Sin embargo, esta tendencia cambia, cuando la mayoría (excepto 
D4) está en desacuerdo al plantear que los conocimientos científicos reconocidos y legitimados universalmente puedan cambiar.

\subsection{ANÁlisis PREGUNTA ABIERTA SOBRE LA DIMENSIÓN} NATURALEZA DE LAS CIENCIAS

En este punto, se expondrán los resultados de análisis de contenido acerca de las respuestas de los docentes de la dimensión de Naturaleza de las Ciencias. La pregunta orientadora de la reflexión en este campo, cuyo propósito era complementar los resultados del cuestionario se planteó en los siguientes términos: ¿Cuál es el papel de los estudios sobre la naturaleza de la ciencia para el profesorado de Química?

Una parte importante del grupo de docentes expresa ideas cercanas a una concepción de la naturaleza de las ciencias de carácter racionalista moderada, considerando que es un aporte al proceso de enseñanza aprendizaje.

El rol fundamental radica en que a partir del conocimiento de la naturaleza de las ciencias se construyen las bases conceptuales y epistemológicas necesarias para un proceso enseñanza-aprendizaje eficaz (D2).

...Bajo todo ese escenario el docente debe permitir a sus estudiantes ir construyendo sus aprendizajes en torno a la ciencia escolar (D1).

Según los docentes existe una necesidad de formar un marco teórico que permita la reflexión y las mejoras de sus prácticas educativas. Por otro lado, hay docentes que manifiestan que luego de participar de los talleres de formación continua, se dan cuenta de la importancia de la comprensión de la naturaleza de las ciencias y como un hecho relevante para la toma de decisiones dentro de sus prácticas pedagógicas: 
El que el profesorado de química conozca los estudios sobre la naturaleza de la ciencia abre oportunidades para un enfoque más profundo, real y complejo sobre el conocimiento que emerge del desarrollo científico, sus implicancias históricas, limitaciones y también aspectos didácticos sobre la enseñanza de la ciencia en el aula (D3).

Una adecuada comprensión de la naturaleza de las Ciencias permite apreciar el valor de las Ciencias, sus alcances y sus límites, que permitirá la toma de decisiones a la hora de planificar las estrategias para los estudiantes. Por esto, se hace necesario, tanto en la formación inicial del docente como en la búsqueda de las instancias de reflexión en comunidades de aprendizaje (D2).

De acuerdo a estos planteamientos, se interioriza los beneficios de la formación continua como eje para la enriquecer el discurso del docente y que se plasma en sus planificaciones y las nuevas estrategias usadas en sus aulas. También es importante el rol que le dan los docentes a la naturaleza de las ciencias como facilitador de aprendizaje de los alumnos:

La naturaleza de las ciencias entrega conocimientos sobre el proceso de construcción del conocimiento científico, ayudando a los profesores de química a comprender este proceso, orientando sus prácticas en determinadas competencias científicas que el currículum pretende alcanzar y que, con los estudios realizados, el profesorado puede tener una base teórica para sustentar sus prácticas (D5).

Es muy importante, porque el estudio sobre la naturaleza de las ciencias permite centrar la enseñanza no sólo en el producto de la ciencia como son las teorías, leyes, etc., sino también en el proceso de cómo se desarrolla el trabajo científico, lo que permite el desarrollo de habilidades y actitudes propias de este quehacer (D4).

272 | INTEREDU № 5 VoL. II (DiCIEMBRE 2021) PÁGs. 245-286. ISSN: 2735-6523 
Contribución de un programa de formación continua a las concepciones epistemológicas en docentes de química

Cuando el profesor sabe qué noción de naturaleza es la que desea transmitir, trabaja para ella. Es la meta hacia la cual se mueve, planificando y actuando intencionadamente, permitiéndole a sus estudiantes comprender la ciencia como un ámbito que trasciende a todas las áreas de su vida y no sólo a una reducida definición empírica (D6).

A partir de lo planteado por los docentes que formaron parte del estudio, se resalta la importancia de un referente teórico, considerando a la naturaleza de las ciencias como eje epistemológico para el desarrollo de sus clases.

\subsection{ANÁLISIS GRUPOS FOCALES SOBRE LA DIMENSIÓN NATURALEZA DE} LAS CIENCIAS

Durante el desarrollo de este estudio se realizaron dos grupos focales donde se analizaron diversas temáticas que se fueron trabajando en los talleres de formación docente. Esto contribuyó al logro del objetivo planteado y referido a las concepciones epistemológicas sobre la Naturaleza de las Ciencias de los docentes.

Sobre el análisis descriptivo de los resultados obtenidos en los grupos focales, se constatan cambios en el discurso de los profesores participantes entre ambos grupos focales. Los docentes muestran una tendencia a preocuparse de que sus planificaciones sean capaces de promover un conocimiento de calidad y una buena formación de sus estudiantes. También surge un creciente interés en el hecho de que los talleres de formación continua les permitan tener una base epistemológica, un referente teórico, para una mejor comprensión de la naturaleza de las ciencias que les proporcione un sustento para enfrentar los problemas de enseñanza que se dan en el aula.

De igual forma, se menciona durante la ejecución de los talleres de formación continua, que al momento de ir desarrollando las habilidades de pensamiento científico en sus estudiantes, se han ido derribando los INTEREDU № 5 VOL. II (DicIEMBRE 2021) PÁGS. 245-286. ISSN: 2735-6523| 273 
temores presentes en un principio y se potencia el entusiasmo y autonomía en el aula. Se fortalece la necesidad de formar un marco teórico que permita la reflexión y la mejora de las prácticas educativas, así como el cambio del discurso del docente frente a sus estudiantes.

Por último, existe un convencimiento por parte de los docentes, de los beneficios de la formación continua como eje para enriquecer el discurso teórico del profesor y que se plasma en sus planificaciones, su motivación y las nuevas estrategias usadas en sus aulas.

La siguiente Figura relaciona las concepciones epistemológicas interpretadas a partir de las declaraciones de los docentes participantes y las categorías propuestas en esta investigación.

Figura 1. Relación entre las concepciones de los docentes participantes y las categorías determinadas.

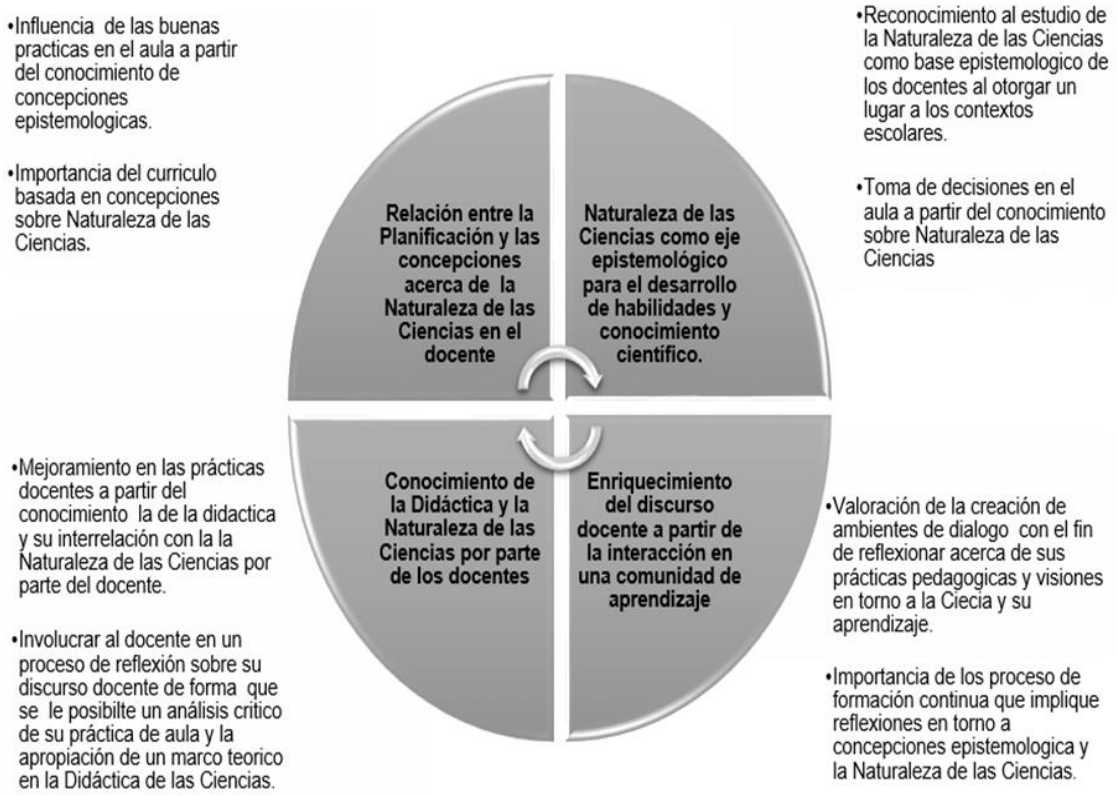

Fuente: Elaboración propia.

274 | INTEREDU № 5 VoL. II (DiCIEMBRE 2021) PÁGs. 245-286. ISSN: 2735-6523 


\section{DISCUSIÓN Y CONCLUSIONES}

De acuerdo a los objetivos propuestos en el estudio, se pueden desarrollar discusión y conclusiones que se plantean a continuación.

Objetivo específico 1. Identificar las concepciones epistemológicas acerca de la naturaleza de las ciencias en docentes de química que se desempeñan en Educación Media.

En relación a este objetivo, debemos señalar que este estudio ha permitido recalcar la importancia de conocer las posturas epistemológicas de los docentes con el fin de mejorar la enseñanza de las ciencias. De tal manera que los cursos de formación continua de los docentes deben favorecer el conocimiento de los diferentes enfoques epistemológicos y entregar a los profesores una visión apropiada de la naturaleza de las ciencias. Adúriz-Bravo (2006), señala que la incorporación de la epistemología en la labor de los docentes de ciencia exige de ellos unos saberes teóricos y prácticos que no siempre han estado presentes en su formación inicial y menos en su formación a través de los años de ejercicios de la profesión. Este autor enfatiza que, al momento de enseñar ciencias, los contenidos epistemológicos pueden fundamentar y dar estructura a las imágenes de ciencia que se consideran actualmente contenidos necesarios para la educación del "ciudadano científicamente alfabetizado".

En relación a la naturaleza de las ciencias, se debe subrayar la incertidumbre en las propias concepciones de los docentes que manifiestan el origen de las ciencias a partir de las demandas y necesidades de la sociedad, afectada por factores económicos, culturales y sociales. Este tipo de factores pueden influir directa o indirectamente en el aprendizaje de las Ciencias. 
Objetivo específico 2. Comparar las concepciones epistemológicas acerca de la Naturaleza de las Ciencias de los docentes antes y después de la participación en talleres de formación continua.

Los resultados sugieren que la mayoría de los docentes participantes de la investigación evidencian una transición desde una visión dogmático positivista a racional moderado, luego de los talleres de formación continua donde se revisaron temáticas relacionadas con concepciones epistemológicas y naturaleza de las ciencias, entre otros. Además de los procesos de reflexión que se realizaron en el seno de la comunidad de aprendizaje. Sin embargo, llama la atención que aún exista una noción positivista de la construcción del conocimiento en los docentes, al considerar que la ciencia es rigurosa y se basa en un método científico, así como también considerar que el docente es el que enseña el conocimiento verdadero e incuestionable. Posiblemente, tales nociones son consecuencia de coexistencia de visiones epistemológicas y de continuar un camino trazado por exigencia del currículum centralizado y de la unidad educativa de cada docente. Esto sucede de manera más frecuente de lo que sería deseable y, como describe Porlán (1999), podemos encontrar que:

...un profesor puede considerar deseable que sus alumnos participen activamente en la clase para mejorar la calidad de su aprendizaje (principio consciente) y, simultáneamente, obstaculizar el cumplimiento de este deseo -porque ha vuelto rutinaria- una respuesta represiva cuando hablan y cuando consigue generar un debate en clase, lo interrumpe dando la formulación acabada de los contenidos que surgen a raíz de dicho debate, pues, aunque no sepa muy bien por qué (teoría 
Contribución de un programa de formación continua a las concepciones epistemológicas en docentes de química implícita), considera que es imprescindible que los alumnos, al margen de que lo olviden o no lo comprendan, han de recibir del profesor el conocimiento que considera correcto ( $p .48$ ).

Tamayo et al. (2009), señalan que superar estos obstáculos por parte de los docentes, ayudará a los estudiantes a mejorar la comprensión de los conocimientos científicos que se discuten en el aula, con el objetivo de dar sentido y valorar los productos de la ciencia y tecnología, para así consecutivamente favorecer espacios de participación socio científica para la toma de decisiones.

Objetivo específico 3. Interpretar cómo las concepciones epistemológicas acerca de la Naturaleza de las Ciencias cambian a partir de la participación de los docentes en talleres de formación continua en el mejoramiento de sus prácticas docentes.

Es destacable el consenso en la implementación de talleres de formación continua donde se den instancias de reflexión docente, que permitan una movilización de las concepciones epistemológicas, sobre naturaleza de las ciencias, en conformidad con la educación científica que necesitan los estudiantes y la sociedad de hoy. El cambio en el discurso del docente permitirá un mayor cuestionamiento de los conocimientos considerados históricamente como válidos, rígidos e incuestionables. Mora y Parga (2008), señalan que los docentes que reflexionan en grupo sobre sus prácticas contribuyen a su desarrollo profesional didáctico y mejoran su discurso al verse reflejados en las experiencias y visiones de otros profesores.

Queda claro que la formación continua en docentes debe realizarse a partir de un trabajo colectivo, que implique el análisis crítico de las propias prácticas de aula y que provoque cierta inquietud, activando el INTEREDU № 5 VoL. II (DiCIEMBRE 2021) PÁGs. 245-286. ISSN: 2735-6523| 277 
cambio didáctico que les permita apropiarse de los componentes teóricos de la didáctica de las ciencias. Es evidente que estas instancias promueven una adecuada planificación (con sus objetivos y actividades) a desarrollar por los docentes, desde una fundamentación con base teórica, que orienta a repensar desde nuevas perspectivas la ciencia que se concibe y se enseña en las aulas. Por ello, se recalca que los talleres de formación continua entreguen un sustento epistemológico a partir de la naturaleza de las ciencias, promoviendo la reflexión docente y su aplicación en la práctica profesional.

Es claro que el aprendizaje que involucre concepciones epistemológicas vinculadas a la naturaleza de las ciencias debe llevar al docente a la permanente reflexión sobre la trascendencia que esto tiene en sus estudiantes y al desarrollo de disciplinar en el aula, así como a otros ámbitos de su vida. El desafío que se plantea es una mayor propuesta de comunidades de aprendizaje que sean el lugar para la consolidación e implementación de un marco teórico y metodológico, que impulse la reflexión en torno a las concepciones epistemológicas del profesorado sobre la alfabetización científica y el docente tome su rol como transformador de los procesos de cambio educativo en el aula.

La principal limitación surgida en este estudio fue la relacionada con el recargado trabajo de los docentes. En relación a las proyecciones debemos mencionar que, sin duda, estos resultados no son generalizables, pero sí son comunes a otros casos de investigación (Rodríguez y López, 2006; Quintanilla, 2006a, Quintanilla, 2006b; Ravanal y Quintanilla, 2010). Por lo tanto, aporta nuevas perspectivas a una mayor participación de los docentes en talleres de formación continua y por ende a comunidades de aprendizaje. Estas nuevas perspectivas les permitirán conocer y comprender sus concepciones epistemológicas, así como debatir y diseñar 278 | INTEREDU № 5 VOL. II (DicieMBRE 2021) PÁGs. 245-286. ISSN: 2735-6523 

docentes de química

nuevas estrategias de intervención. Esto facilitará el desarrollo hacia un conocimiento profesional sustentando por un marco teórico efectivo que contribuya a las decisiones de un diseño didáctico y en los ambientes de aprendizaje de la química escolar.

\section{REFERÊNCIAS BIBLIOGRÁFICAS}

Abela, A. (2002). Las técnicas de análisis de contenido: una revisión actualizada. Centro de estudio andaluz.

Acevedo, J. (2004). Reflexiones sobre las finalidades de la enseñanza de las ciencias: educación científica para la ciudadanía. Revista Eureka sobre Enseñanza y Divulgación de las Ciencias, 1(1), 3-16. https://www.redalyc.org/pdf/920/92010102.pdf

Acevedo, J. (2010). Formación del profesorado de Ciencias y Enseñanza de la naturaleza de la Ciencia. Revista Eureka sobre Enseñanza y Divulgación de las Ciencias, 7(3), 653-660.

http://dx.doi.org/10.25267/Rev_Eureka_ensen_divulg_cienc.2010.v7.i3.04 Adúriz-Bravo, A. (2004). Apuntes sobre la formación epistemológica de los profesores de ciencias naturales. Pedagogía y Saberes, (21), 9-19. https://doi.org/10.17227/01212494.21pys9.19

Adúriz-Bravo, A. (2006). La epistemología en la formación de profesores de ciencias. Revista Educación y Pedagogía, 18(45), 25-36.

https://revistas.udea.edu.co/index.php/revistaeyp/article/view/6084

Adúriz-Bravo, A. (2005). ¿Qué naturaleza de la ciencia hemos de saber los profesores de ciencias? Una cuestión actual de la investigación didáctica. [Número extraordinario]. Tecné, Episteme y Didaxis, 23-33.

Adúriz-Bravo, A., e Izquierdo, M. (2002). Acerca de la didáctica de las ciencias como disciplina autónoma. Revista Electrónica de Enseñanza de las Ciencias, $1(3)$, 130-140. http://reec.uvigo.es/volumenes/volumen1/REEC_1_3_1.pdf INTEREDU № 5 VOL. II (DiCiembre 2021) PÁGs. 245-286. ISSN: 2735-6523| 279 
Aiello, M. (2004). Concepciones epistemológicas del docente y su incidencia en la enseñanza de las ciencias. Revista Colombiana de Educación, (47), 1-28. https://doi.org/10.17227/01203916.5520

Aigneren, M. (2002). La técnica de recolección de información mediante grupos focales. La Sociología en sus Escenarios, (6), 1-32. https://revistas.udea.edu.co/index.php/ceo/article/view/1611/1264

Alarcón, N. (2018). La enseñanza de la epistemología y estrategias de abordaje para el aula. Historia Regional, (38), 1-10. http://historiaregional.org/ojs/index.php/historiaregional/article/vi ew/232/510

Alburquerque, V. (2016). Epistemología de las Ciencias Naturales. Universidad de Valladolid. Facultad de Ciencias. http://uvadoc.uva.es/handle/10324/19313

Aldana, M. (2008). Enseñanza de la investigación y epistemología de los docentes. Educación y Educadores, 11(2), 61-68. http://www.scielo.org.co/pdf/eded/v11n2/v11n2a04.pdf

Alonso, A. (2000). Creencias del profesorado sobre la naturaleza de la ciencia. Revista Interuniversitaria de Formación del Profesorado, (37), 18720. https://dialnet.unirioja.es/servlet/articulo?codigo $=118066$

Angulo, F. (2002). Formulación de un modelo de autorregulación de los aprendizajes desde la formación profesional del biólogo y del profesor de biología. [Tesis Doctoral]. Universidad Autónoma de Barcelona.

Bisquerra, R., y Alzina, R. B. (2004). Metodología de la investigación educativa (Vol. 1). Editorial, La Muralla.

Briscoe, C. (1991). The Dynamic interactions among beliefs, role metaphors, and teaching practices: A case study of teacher change. Science Education.

Camacho, J. P. (2013). Concepciones sobre ciencia y género en el Profesorado de química: aproximaciones desde un estudio colectivo de casos. Ciência \& 280 | INTEREDU № 5 VoL. II (DiCIEMBRE 2021) PÁGs. 245-286. ISSN: 2735-6523 
Contribución de un programa de formación continua a las concepciones epistemológicas en docentes de química Educação, 19(2), 323-338. https://doi.org/10.1590/S151673132013000200007

Carvajal, E. y Gómez, R. (2002). Concepciones y representaciones de los maestros de secundaria y bachillerato sobre la naturaleza, el aprendizaje y la enseñanza de las ciencias. Revista Mexicana de Investigación Educativa, 7(16), 577-602.

https://www.redalyc.org/pdf/140/14001607.pdf

Cuellar, L. (2010). La historia de la química en la reflexión sobre la práctica profesional docente. Un estudio de caso desde la enseñanza de la Ley Periódica. [Tesis Doctoral]. Pontificia Universidad Católica de Chile. http://www7.uc.cl/sw_educ/educacion/grecia/plano/html/pdfs/bibl ioteca/DOCTOR/TesisDocLC.pdf

Eirín-Nemiña, R. (2018). Las comunidades de aprendizaje como estrategia de desarrollo profesional de docentes de Educación Física. Estudios Pedagógicos (Valdivia), 259-278. http://dx.doi.org/10.4067/S0718-07052018000100259

Fernández, M. T., Bertrán, A. M. T., Ibarra, R. E. P., y Pacheco, A. C. L. (2009). Concepciones de los maestros sobre la enseñanza y el aprendizaje y sus prácticas educativas en clases de Ciencias Naturales. Enseñanza de las Ciencias: Revista de Investigación y Experiencias Didácticas, 27(2), 287-298. https://core.ac.uk/download/pdf/13279305.pdf

García-Carmona, A. (2012). Cómo enseñar Naturaleza de la Ciencia (NDC) a través de experiencias escolares. Alambique: Didáctica de las Ciencias Experimentales, (72), 55-63.

https://idus.us.es/bitstream/handle/11441/59541/Como_ense\%F1ar _Naturaleza_de_la_Ciencia_NDC_a_traves_de_experiencias_escol ares_de_investigacion.pdf?sequence $=1$

Hernández, R., y Torres, M. (2018). Metodología de la investigación (Vol. 4). McGraw-Hill Interamericana. 
Izquierdo, M. (2005). Hacia una teoría de los contenidos escolares. Enseñanza de las Ciencias: Revista de Investigación y Experiencias Didácticas, 23(1), 111-122. https://core.ac.uk/download/pdf/38990162.pdf

Juan, S., y Roussos, A. (2010). El focus group como técnica de investigación cualitativa (informe n⿳254). Departamento de Investigaciones Area de Psicología Clínica Serie Métodos de Psicología Clínica № 9. http://repositorio.ub.edu.ar/bitstream/handle/123456789/4781/254_ Roussos.pdf?sequence $=1 \&$ isAllowed $=\mathrm{y}$

Kuhn, T. S. (2019). La estructura de las revoluciones científicas. Fondo de Cultura Económica.

Lakatos, I. (1983). La metodología de los programas de investigación científica. Alianza.

Lederman, N. G. (2006). Research on nature of science: reflections on the past, anticipations of the future. Asia-Pacific Forum on Science Learning and Teaching, 7(1), 1-11. https://www.eduhk.hk/apfslt/v7_issue1/foreword/index.htm

Martínez, C. (2015). Concepciones y prácticas docentes e investigativas del profesorado universitario de ciencias: Un estudio de la enseñanza de la Biología. [Tesis doctoral]. Universitat de Barcelona. https://www.tdx.cat/bitstream/handle/10803/294034/CMG_TESIS.pdf;js essionid=E2ECFADBAE13070BC94C5D2D37FA2430? sequence $=1$

Martínez, C., y González, C. (2014). Concepciones del profesorado universitario acerca de la ciencia y su aprendizaje y cómo abordan la promoción de competencias científicas en la formación de futuros profesores de Biología. Enseñanza de las Ciencias: Revista de Investigación y Experiencias Didácticas, 32(1), 51-81. https://raco.cat/index.php/Ensenanza/article/view/287508 
Ministerio de Educación (2017). Orientaciones para la apropiación de las bases curriculares de $7^{\underline{0}}$ básico a $2^{\underline{o}}$ medio. Ministerio de Educación Chile. https://media.mineduc.cl/wpcontent/uploads/sites/28/2017/05/Orientacio nes-apropiacion-BC-7\%C2\%BA-2\%C2\%BAM-web corregido.pdf

Mora, P. W. M., y Parga, L. D. L. (2008). El conocimiento didáctico del contenido en química: integración de las tramas de contenido históricoepistemológicas con las tramas de contexto-aprendizaje. Tecné Episteme y Didaxis: TED, (24), 56-81. https://doi.org/10.17227/ted.num241083

Organización para la Cooperación y Desarrollo Económico OECD (2006). Marco de la evaluación. Conocimiento y habilidades en Ciencias, Matemáticas y Lectura. OECD. https://www.oecd.org/

Pavéz, K., y Pérez, O. (2013). Las visiones de alfabetización científica del profesorado y su relación con la práctica de aula. [Tesina para optar al título de Profesor de Biología y Ciencias Naturales]. Universidad Metropolitana de Ciencias de la Educación, Chile.

Popper, K. (1962). La lógica de la investigación científica. Madrid. Tecnos.

Porlán Ariza, R., Rivero García, A., y Martín del Pozo, R. (1998). Conocimiento profesional y epistemología de los profesores, II: estudios empíricos y conclusiones. Enseñanza de las Ciencias. Revista de Investigación y Experiencias Didácticas, 16(2), 271-288. https://raco.cat/index.php/Ensenanza/article/view/21534

Porlán, R. (1999). Investigar la práctica. Cuadernos de Pedagogía, (276), 48-49. Quintanilla, M. (2006). La ciencia en la escuela: un saber fascinante para aprender a "leer el mundo". Revista Pensamiento Educativo, 39(2), 177-204. http://pensamientoeducativo.uc.cl/index.php/pel/article/view/2389 7/19233

Quintanilla, M., Astroza, V., De la Fuente, R., Camacho, J., y Cuellar, L. (2006a). Imagen de las metaciencias en la formación inicial de profesores 
de EGB. Facultad de Educación, Pontificia Universidad Católica de Chile. Santiago de Chile.

Quintanilla, M., Labarrere, A., Santos, M., Cádiz, J., Cuellar, L., Saffer, G., y Camacho, J. (2006b). Elaboración, validación y aplicación preliminar de un cuestionario sobre ideas acerca de la imagen deficiencia y Educación. Grupo GRECIA Departamento de Didáctica, Facultad de Educación Pontificia Universidad Católica de Chile. http://www7.uc.cl/sw_educ/educacion/grecia/plano/html/pdfs/line a_investigacion/Que_Ciencia_Ensenar_IEC/IEC_045.pdf

Quintanilla, M., y Ravanal, L. E. (2009). Racionalidades epistemológicas y didácticas del profesorado de Biología sobre la enseñanza y aprendizaje del metabolismo: aportes para el debate de una nueva clase de ciencias. [Tesis Doctoral]. Universidad Academia de Humanismo Cristiano.

Quintanilla, M., Joglar, C., Jara, R., Camacho, J., Ravanal, E., Labarrere, A., Cuellar, L., Izquierdo, M. y Chamizo, J. (2010). Resolución de problemas científicos escolares y promoción de competencias de pensamiento científico. ¿Qué piensan los docentes de Química en ejercicio? Enseñanza de las Ciencias, 28(2), 185-198. https://raco.cat/index.php/Ensenanza/article/view/199612

Ravanal, E., Quintanilla, M., y Labarrere, L. (2012). Concepciones epistemológicas del profesorado de biología en ejercicio sobre la enseñanza de la biología. Ciência \& Educação, 18(4), 875-895. https://doi.org/10.1590/S1516-73132012000400009

Ravanal, E., y Quintanilla, M. (2010). Caracterización de las concepciones epistemológicas del profesorado de Biología en ejercicio sobre la naturaleza de la ciencia. Revista Electrónica de Enseñanza de las Ciencias, 9(1), 111124. http://reec.uvigo.es/volumenes/volumen9/ART7_VOL9_N1.pdf 

docentes de química

Rodríguez, D. P., y López, Á. D. (2006). ¿Cómo se articulan las concepciones epistemológicas y de aprendizaje con la práctica docente en el aula? Tres estudios de caso de profesores de secundaria. Revista Mexicana de Investigación Educativa, 11(31), 1307-1335.

http://www.scielo.org.mx/scielo.php?script=sci_abstract\&pid=S140566662006000401307\&lng=es\&nrm=iso

Salazar, R., y Serrano, S. (2017). Concepciones epistemológicas de los docentes sobre los ejes integradores. Conocimiento, naturaleza y funciones. Acción Pedagógica, 26(1), 96-104.

https://dialnet.unirioja.es/servlet/articulo?codigo $=6344977$

Sánchez, C., y Gómez, R. (2013). Enseñanza de las ciencias naturales para el desarrollo de competencias científicas. Amazonia Investiga, 2(3), 30-53. https://amazoniainvestiga.info/index.php/amazonia/article/view/646/607 Solbes, J., y Torres, N. (2013). Concepciones y dificultades del profesorado sobre el pensamiento crítico en la enseñanza de las ciencias. Enseñanza de las Ciencias, (Extra), 3389-3393.

https://dialnet.unirioja.es/servlet/articulo?codigo=6872670\&orden= 0\&info=link

Tamayo, Ó., y Orrego, M. (2009). Aportes de la naturaleza de la ciencia y del contenido pedagógico del conocimiento para el campo conceptual de la educación en ciencias. Revista Educación y Pedagogía, 17(43), 9-25. https://revistas.udea.edu.co/index.php/revistaeyp/article/view/6051

Tobin, K., y Campbell, M. (1997). Beliefs about the Nature of Science and the Enacted Science Curriculum. Science \& Education, 6, 355-371. https://doi.org/10.1023/A:1008600132359

Toulmin, S. (1977). La comprensión humana I. El uso colectivo y la evolución de los conceptos. Madrid Alianza.

Vildósola, T (2017). El conocimiento sobre la epistemología de la ciencia como eje para mejorar la relación investigación-práctica en la formación 
Sandra Villegas y Luigi Cuellar

inicial docente en ciencias: el caso de Chile. Enseñanza de las Ciencias: Revista de Investigación y Experiencias Didácticas, (Extra), 89-96. https://dialnet.unirioja.es/servlet/articulo?codigo $=6690011$

286 | INTEREDU № 5 VOL. II (DiCIEMBRE 2021) PÁGS. 245-286. ISSN: 2735-6523 